\title{
La tuberculose au Canada : 2017
}

\author{
M LaFreniere ${ }^{1}, \mathrm{H}_{\text {Hussain }}{ }^{1,2}, \mathrm{~N} \mathrm{He}^{1,2}, \mathrm{M} \mathrm{McGuire}^{1}$
}

\section{Résumé}

Contexte : La tuberculose (TB) est un problème de santé majeur qui a touché environ 10 millions de personnes dans le monde en 2017. L'Agence de la santé publique du Canada (ASPC) surveille la TB active au moyen d'un système de surveillance national, en collaboration avec les provinces et les territoires.

Objectif : Présenter un résumé épidémiologique des cas de TB active déclarés en 2017. Les résultats sont discutés dans le contexte des données de l'année précédente. Les résultats du traitement pour les cas diagnostiqués en 2016 sont également présentés.

Méthodologie : Le Système canadien de déclaration des cas de tuberculose est un système de surveillance contenant des données non nominales sur les cas de tuberculose active au Canada. Les données sont recueillies annuellement par les provinces et territoires, analysées par l'ASPC et validées par chaque province et chaque territoire.

Résultats : Au Canada, 1796 cas de TB active ont été déclarés en 2017, comparativement à 1750 en 2016, ce qui représente une augmentation de 2,6\%. De même, le taux d'incidence est passé de 4,8 à 4,9 pour 100000 habitants. Les personnes nées à l'étranger continuaient de représenter la majorité $(71,8 \%$ ) des cas déclarés, mais le taux d'incidence chez les Autochtones nés au Canada est resté le plus élevé avec 21,5 cas pour 100000 habitants. Le taux était particulièrement élevé chez les Inuit (205,8 pour 100000 habitants). Comme pour la décennie précédente, les taux d'incidence de la TB en 2017 sont plus élevés chez les hommes (5,5 pour 100000$)$ que chez les femmes (4,3 pour 100000$)$, et la majorité des cas $(45,6 \%)$ ont été observés chez des personnes de 15 à 44 ans. Le taux d'incidence était le plus élevé chez les adultes de plus de 75 ans (13,8 cas pour 100000 habitants chez les hommes et 7,2 chez les femmes). Parmi les cas de TB diagnostiqués en 2016 pour lesquels des résultats ont été déclarés, 80,4\% ont été traités avec succès.

Conclusion : Bien que le taux d'incidence de la TB en 2017 au Canada soit demeuré faible par rapport au reste du monde et qu'il soit demeuré relativement stable au cours de la dernière décennie, le nombre de cas et le taux augmentent graduellement depuis 2014. Les Canadiens autochtones et ceux qui sont nés à l'étranger continuent d'être représentés de manière disproportionnée parmi les cas de TB. Les données provenant de la surveillance de la TB au Canada sont une source d'information importante pour surveiller les progrès et éclairer les mesures de santé publique visant à alléger le fardeau de la TB au Canada, l'objectif final étant de l'éliminer complètement.
Cette oeuvre est mise à la disposition selon les termes de la licence internationale Creative Commons Attribution 4.0

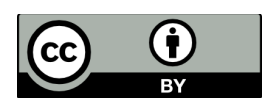

Affiliations

${ }^{1}$ Centre de la lutte contre les maladies transmissibles et les infections, Agence de la santé publique du Canada, Ottawa (Ontario)

2 École de santé publique Dalla Lana, Université de Toronto, Toronto (Ontario)

*Correspondance: phac. tb.surveillance.aspc@canada.ca

Citation proposée : LaFreniere M, Hussain H, He N, McGuire M. La tuberculose au Canada : 2017. Relevé des maladies transmissibles au Canada. 2019;45(2/3):73-80. https://doi.org/10.14745/ccdr. v45i23a04f

Mots-clés : tuberculose, surveillance, taux d'incidence 


\section{Introduction}

La tuberculose (TB) est l'une des maladies infectieuses les plus courantes et l'une des principales causes de décès dans le monde. L'Organisation mondiale de la santé (OMS) a estimé à 10 millions le nombre de nouveaux cas de TB dans le monde en 2017 (1). Dans le cadre de sa Stratégie pour mettre fin à la tuberculose, I'OMS a rédigé un cadre d'action pour l'élimination de la TB dans les pays à faible incidence (Towards TB Elimination: An Action Framework for Low-Incidence Countries), c'est-à-dire les pays dont le taux d'incidence est au plus de 10 cas de TB pour 100000 habitants. Dans ce document, l'OMS propose des moyens de poursuivre la réduction des taux de TB de façon à atteindre, d'ici 2035, un niveau équivalant à la définition d'élimination totale, soit 0,1 cas pour 100000 habitants (2).

Bien que le Canada soit un pays à faible incidence de TB, les taux d'incidence de la TB sont systématiquement supérieurs au seuil de faible incidence dans certaines sous-populations du pays, notamment les personnes nées à l'étranger et les Canadiens autochtones (3). On note une forte activité de la TB dans le Nord canadien depuis de nombreuses années, en particulier chez les Inuit $(3,4)$. La TB chez les Canadiens nés à l'étranger représente également un lourd fardeau au Canada (3). Statistique Canada prévoit des taux de croissance élevés dans ces deux populations au Canada par rapport à la population canadienne dans son ensemble $(5,6)$. Il est donc particulièrement important de diagnostiquer et de traiter la TB, à la fois pour éliminer les effets d'une TB active sur les personnes atteintes et pour empêcher toute propagation ultérieure.

Au Canada, l'Agence de la santé publique du Canada (ASPC), en partenariat avec les provinces et les territoires, assure la surveillance nationale des nouveaux cas de TB active et des cas de retraitement. L'objectif principal du Système canadien de déclaration des cas de tuberculose (SCDCT), le système national de surveillance des cas au Canada, est de surveiller et de signaler le nombre de cas et les taux de TB active au Canada. La déclaration annuelle des cas de TB dans l'ensemble du pays est importante pour mieux comprendre l'épidémiologie de la TB au Canada au fil du temps, pour éclairer les mesures de santé publique et pour suivre les progrès accomplis au Canada dans la réduction de l'incidence de la TB, en vue de son élimination totale (7).

Le présent rapport vise à présenter un aperçu descriptif des cas de TB au Canada en 2017 selon l'âge, le sexe, l'origine, la province ou le territoire et la classification du diagnostic, en comparant ces données à celles des années précédentes. On y présente également les résultats des traitements pour les cas de TB signalés dans le SCDCT en 2016.

\section{Méthodologie}

Le SCDCT contient des données non nominales sur les personnes chez qui on a diagnostiqué une TB active au Canada. Les méthodes du SCDCT, notamment les processus de collecte de données, la gestion des données, le contrôle et l'analyse de la qualité des données, ainsi que la classification et la catégorisation des sous-groupes de population, sont expliquées en détail ailleurs (8). En résumé, les autorités de santé publique des provinces et des territoires soumettent volontairement des données sur tous les cas nouveaux ou de retraitement de la TB active qui répondent à la définition canadienne pour la surveillance nationale (8). Les données sur les résultats du traitement sont soumises de 12 à 18 mois après la soumission du rapport de cas initial. Si le traitement est en cours au moment de la soumission des données à I'ASPC, la province ou le territoire qui effectue la déclaration soumet un rapport provisoire, qui est suivi de mises à jour annuelles jusqu'à la fermeture du dossier. Les données à jour des années précédentes reçues après la soumission initiale sont utilisées dans le rapport le plus récent.

Il y a deux catégories de TB active : la TB de l'appareil respiratoire et la TB non respiratoire. La première comprend la tuberculose pulmonaire, la TB de la plèvre et la TB des ganglions lymphatiques intrathoraciques ou médiastinaux, du larynx, du nasopharynx, du nez et des sinus (9). La maladie se manifeste initialement par un épanchement pleural causé par une infection récente (dans les 24 mois précédents) à Mycobacterium tuberculosis. La TB non respiratoire correspond aux autres formes de la maladie.

Les taux d'incidence dans ce rapport ont été calculés selon le nombre de cas par tranche de 100000 habitants. Les données démographiques utilisées pour calculer ces taux proviennent de différentes sources. Les données sur la population canadienne sont fondées sur des estimations de la population canadienne établies par Statistique Canada en milieu d'année (données non publiées). Les données sur la population née à l'étranger sont basées sur le Recensement du Canada de 2016 (10). Les estimations pour la population des groupes autochtones en 2017, à savoir les Premières Nations, les Métis et les Inuit, sont tirées de l'Enquête nationale auprès des ménages (11). Les données sur la population inscrite des Premières Nations vivant à l'intérieur et à l'extérieur des réserves proviennent du Système d'inscription des Indiens de Relations Couronne-Autochtones et Affaires du Nord Canada et datent du 31 décembre 2017 (données non publiées).

Les données originales ont été conservées conformément à la directive de I'ASPC sur la collecte, I'utilisation et la diffusion de l'information sur la santé publique. Les données ont été nettoyées et analysées avec SAS Enterprise Guide 5.1 et 
Microsoft Excel 2010. Les résultats descriptifs sont présentés ici. Aucune procédure statistique n'a été utilisée pour les analyses comparatives, et aucune technique statistique n'a été appliquée pour tenir compte des données manquantes. II convient de noter que la Colombie-Britannique n'a fourni aucune information en 2017 pour les Autochtones inscrits. Par conséquent, les résultats pour cette province sont classés uniquement selon la population née au Canada et la population née à l'étranger. Des tableaux supplémentaires sont disponibles sur demande (voir la liste des tableaux en annexe).

\section{Résultats}

En 2017, 1796 cas de TB active ont été déclarés au Canada, comparativement à 1750 en 2016, ce qui représente une augmentation de $2,6 \%$. Le taux d'incidence a augmenté en conséquence, passant de 4,8 à 4,9 pour 100000 habitants. Parmi tous les cas signalés en $2017,92,2 \%$ étaient de nouveaux cas de TB active, et $5,3 \%$ étaient des cas de retraitement (au moins un épisode de TB signalé antérieurement). Les antécédents d'infection tuberculeuse étaient inconnus pour 2,5\% des cas signalés. Le nombre de cas de TB et le taux d'incidence au Canada ont légèrement augmenté depuis 2014, lorsque le taux d'incidence était de 4,5 cas pour 100000 habitants. Toutefois, sur une période de onze ans (depuis 2007), le taux d'incidence n'a que très peu augmenté (il était de 4,8 pour 100 000). Le nombre de cas, quant à lui, a quelque peu fluctué depuis 2007, mais le nombre absolu de cas signalés en 2017 (1 796 cas) a augmenté par rapport à 2007 (1575 cas) (figure 1).

Figure 1 : Nombre de cas de tuberculose déclarés et taux d'incidence par année, Canada, 20072017

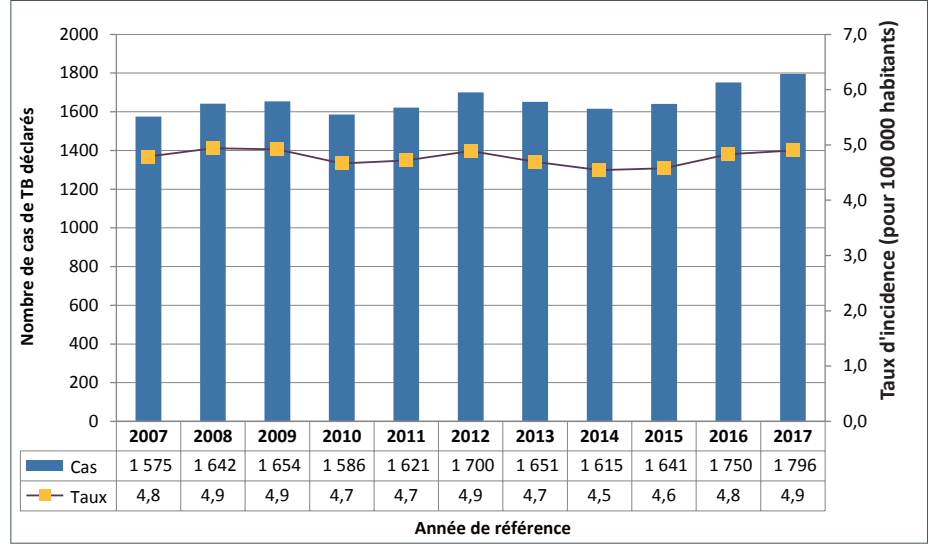

Abréviation : TB, tuberculose

\section{Distribution géographique des cas de tuberculose}

Au Canada, les taux d'incidence de la TB variaient considérablement d'une province et d'un territoire à l'autre en 2017 (tableau 1). Aucun cas de TB n'a été signalé à I'Île-du-Prince-Édouard. Les taux d'incidence étaient inférieurs au taux national de 4,9 cas pour 100000 habitants à Terre-NeuveetLabrador $(2,5)$, en Nouvelle-Écosse $(0,9)$, au Nouveau-Brunswick $(1,1)$, au Québec $(2,6)$ et en Ontario $(4,8)$, mais ils étaient légèrement supérieurs au taux national en Colombie-Britannique $(5,3)$, en Alberta $(5,3)$ et dans les Territoires du Nord-Ouest $(6,7)$. Les taux d'incidence les plus élevés ont été observés en Saskatchewan $(8,1)$, au Manitoba $(14,0)$, au Yukon $(20,8)$ et au Nunavut $(265,8)$. La majorité des cas $(64,4 \%)$ étaient concentrés en Ontario (37,6\%), en Colombie-Britannique $(14,1 \%)$ et en Alberta (12,6\%). Alors que la plupart des provinces et des territoires ont déclaré peu de changement par rapport à 2016, au Nunavut, le nombre de cas a presque doublé de 2016 à 2017 et le taux d'incidence y est passé de 145,6 à 265,8 pour 100000 (tableau 1).

Tableau $1:$ Nombre de cas de tuberculose déclarés et taux d'incidence pour 100000 habitants par province et territoire au Canada, 2016-2017

\begin{tabular}{|l|r|r|r|r|}
\hline \multirow{2}{*}{\multicolumn{1}{|c|}{ Province }} & \multicolumn{2}{|c|}{2016} & \multicolumn{2}{c|}{2017} \\
\cline { 2 - 5 } & \multicolumn{1}{|c|}{ Cas } & \multicolumn{1}{c|}{ Taux } & \multicolumn{1}{c|}{ Cas } & \multicolumn{1}{c|}{ Taux } \\
\hline $\begin{array}{l}\text { Terre-Neuve-et- } \\
\text { Labrador }\end{array}$ & 25 & 4,7 & 13 & 2,5 \\
\hline Île-du-Prince-Édouard & 4 & 2,7 & 0 & 0,0 \\
\hline Nouvelle-Écosse & 3 & 0,3 & 9 & 0,9 \\
\hline Nouveau-Brunswick & 12 & 1,6 & 8 & 1,1 \\
\hline Québec & 252 & 3,0 & 217 & 2,6 \\
\hline Ontario & 641 & 4,6 & 676 & 4,8 \\
\hline Manitoba & 201 & 15,2 & 187 & 14,0 \\
\hline Saskatchewan & 91 & 7,9 & 94 & 8,1 \\
\hline Alberta & 238 & 5,6 & 227 & 5,3 \\
\hline Colombie-Britannique & 225 & 4,7 & 253 & 5,3 \\
\hline Yukon & 1 & 2,7 & 8 & 20,8 \\
\hline Territoires du Nord- & 3 & 6,7 & 3 & 6,7 \\
\hline Ouest & 54 & 145,6 & 101 & 265,8 \\
\hline Nunavut & 1750 & 4,8 & 1796 & 4,9 \\
\hline Total pour le Canada & & & & \\
\hline
\end{tabular}

\section{La tuberculose selon le sexe et l'âge}

Sur les 1796 cas de TB signalés en 2017, 792 (44,1\%) étaient des femmes et 1004 (55,9\%) étaient des hommes, ce qui correspond à un taux d'incidence de 4,3 chez les femmes et de 5,5 chez les hommes par tranche de 100000 habitants. Depuis 2007, les hommes présentent systématiquement une proportion plus élevée de cas de TB et, par conséquent, des taux d'incidence plus élevés (figure 2). 
Figure 2 : Nombre de cas de tuberculose déclarés et taux d'incidence selon le sexe et l'année, Canada, 20072017

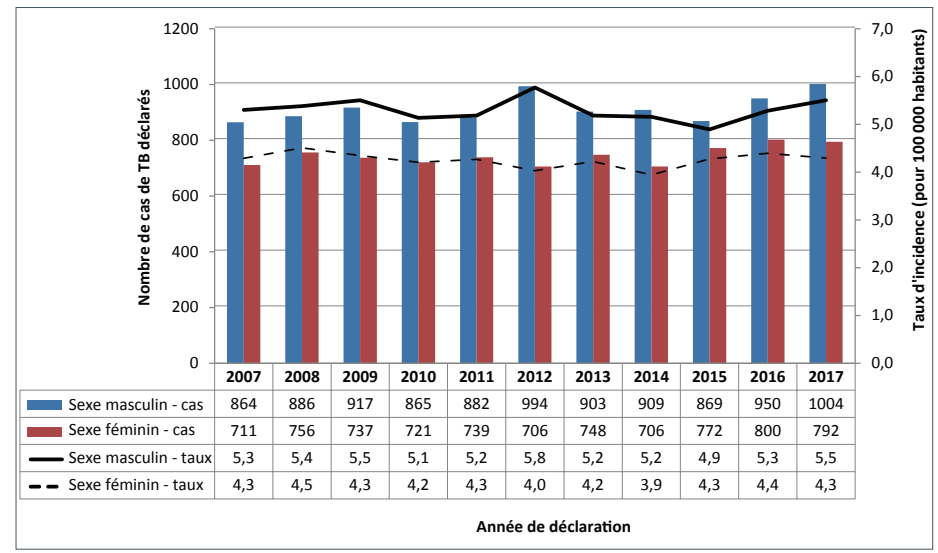

Abréviation : TB, tuberculose

Chez les personnes de 44 ans et moins, les taux d'incidence de la TB pour les hommes et les femmes étaient similaires. Après 44 ans toutefois, l'écart dans les taux d'incidence des hommes et des femmes commence à se creuser. Chez les adultes de 75 ans et plus, les taux d'incidence pour les hommes étaient presque le double de celui des femmes (13,8 cas par rapport à 7,2 cas pour 100000 habitants) (figure 3).

Figure 3 : Taux d'incidence de la tuberculose selon le sexe et l'âge et pourcentage des cas de tuberculose selon le groupe d'âge, Canada, 2017

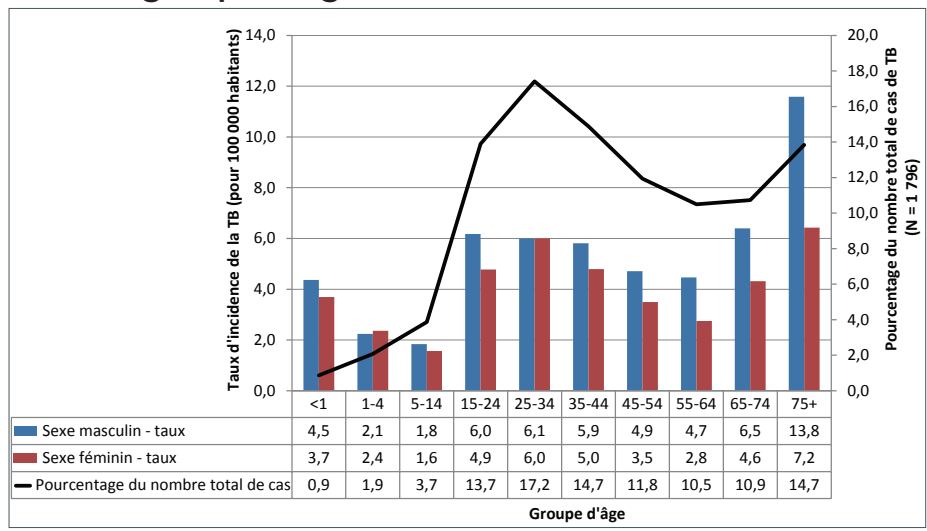

Abréviations : N, nombre total; TB, tuberculose

Les personnes de 75 ans et plus ont le taux d'incidence le plus élevé (10,0 cas pour 100000 habitants), suivies du groupe des 25 à 34 ans (6,0 pour 100 000). La tranche d'âge ayant le taux $d^{\prime}$ 'incidence le plus faible est les 5 à 14 ans (1,7 pour 100 000). Le taux d'incidence chez les enfants de moins d'un an est passé de 2,1 pour 100000 en 2016 à 4,1 pour 100000 en 2017. Le plus grand nombre de cas de TB a été observé chez les 25 à 34 ans ( $n=309 ; 17,2 \%$ du nombre total de cas) (figure 3 ). Les enfants représentaient une faible proportion du nombre total de cas de TB en 2017 : les nourrissons de moins d'un an représentaient $0,9 \%$ du nombre total de cas et les enfants de 1 à 14 ans, 5,6\%. Les tendances dans les cas de TB par tranche d'âge ont peu changé au cours des 10 dernières années (3).

\section{La tuberculose selon l'origine}

Les personnes nées à l'étranger représentaient la majorité des cas de TB déclarés au Canada en 2017 ( $n=1290 ; 71,8 \%$ ), suivies des Autochtones nés au Canada ( $n=313 ; 17,4 \%$ ) et des non-Autochtones nés au Canada ( $n=125 ; 7,0 \%$ ) (figure 4). Dans $1,6 \%$ des cas $(n=28)$, les personnes atteintes étaient nées au Canada, mais le signalement n'en précisait pas davantage, et l'origine était inconnue dans $2,2 \%$ des cas $(n=40)$ (figure 4).

Figure 4: Répartition des cas de tuberculose selon l'origine, Canada, 2017

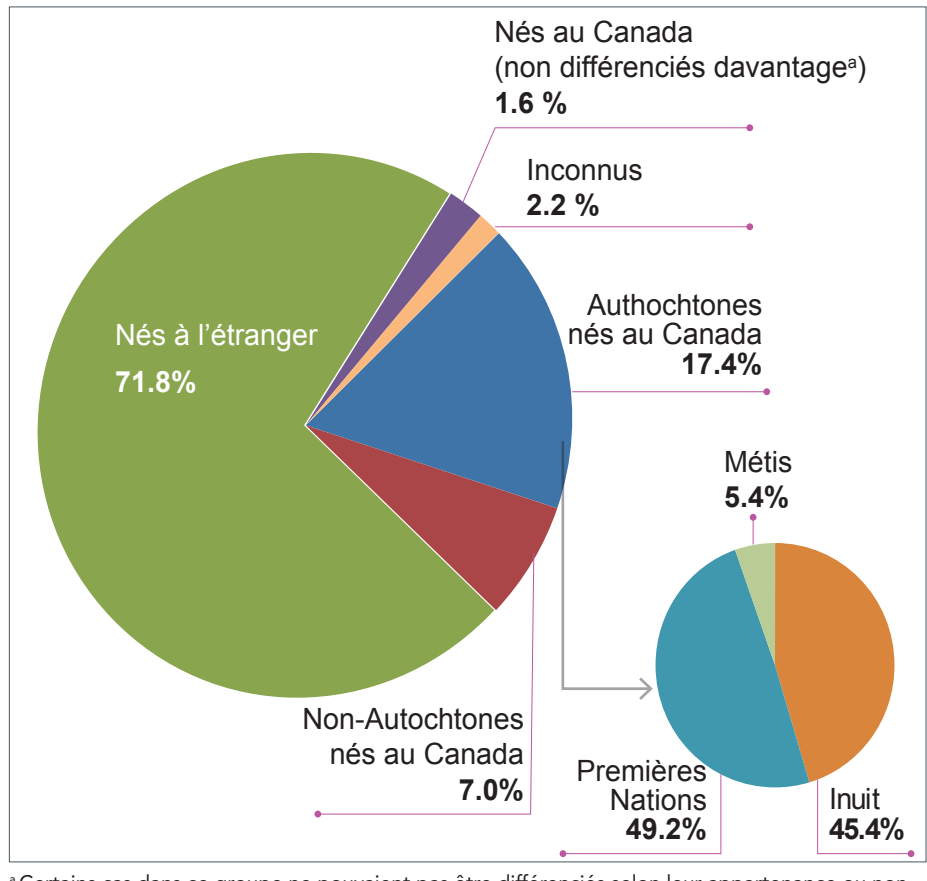

a Certains cas dans ce groupe ne pouvaient pas être différenciés selon leur appartenance ou non au groupe des Autochtones

\section{Cas de tuberculose chez les Autochtones}

En 2017 au Canada, 313 cas de TB ont été déclarés chez les Autochtones nés au Canada, ce qui se traduit par un taux d'incidence de 21,5 cas pour 100000 habitants, soit une légère baisse par rapport à 2016, alors que le taux était de 23,3 cas pour 100000 habitants (tableau 2). Les membres des Premières Nations comptaient pour $49,2 \%(n=154)$ de ces cas, les Inuit, pour 45,4\% $(n=142)$ et les Métis, pour 5,4\% ( $n=17)$ (figure 4). Chez les Métis, le taux d'incidence de la TB était de 3,5 cas pour 100000 habitants, ce qui est inférieur au taux pour l'ensemble du Canada (4,9 par 100 000), mais supérieur à celui des non-Autochtones nés au Canada (0,5 par 100 000). Ce taux a généralement diminué avec le temps, passant de 7,5 cas pour 100000 habitants en 2007 à 2,1 cas en 2016, mais il a affiché une légère augmentation en 2017 (tableau 2). Chez les membres des Premières Nations, le taux d'incidence de la TB était de 17,1 cas pour 100000 habitants en 2017, ce qui est plus bas que le taux de 2016 (23,6 pour 100 000). Ce taux a toutefois fluctué depuis 2007 (tableau 2). Le taux d'incidence de la TB chez les membres inscrits des Premières Nations vivant à dans les réserves en 2017 était de 21,7 pour 100 000, ce qui représente une diminution 
par rapport à l'année précédente (33,9 par 100 000). Le taux $d$ 'incidence chez les membres inscrits des Premières Nations vivant à l'extérieur des réserves a également diminué, passant de 14,5 pour 100000 en 2016 à 9,6 pour 100000 en 2017 (tableau 2). En 2017, 142 cas de TB ont été déclarés chez les Inuits, comparativement à 113 en 2016, ce qui représente une augmentation de $25,7 \%$. Le taux d'incidence correspondant est passé de 168,7 pour 100000 en 2016 à 205,8 pour 100000 en 2017. Les taux d'incidence au sein de la population inuite ont varié d'un plancher de 85,2 par 100000 en 2007, à un plafond de 251,6 pour 100000 en 2012, mais ils sont demeurés systématiquement plus élevés que les taux de tous les autres sous-groupes de la population depuis 2007 (tableau 2).

\section{Cas de tuberculose chez les personnes nées à l'étranger}

À l'instar des années précédentes, les personnes nées à l'étranger formaient le groupe le plus représenté dans les cas de TB au Canada en 2017. En effet, 71,8 \% du nombre total de cas ( $n=1290$ ) touchait des personnes nées à l'étranger, ce qui correspond à un taux d'incidence de 14,7 cas pour 100000 habitants. Bien que le taux d'incidence de la TB chez les Canadiens nés à l'étranger soit demeuré relativement stable depuis 2007 ( $n=14,8$ pour 100000 habitants), le nombre absolu a affiché une croissance constante (tableau 2). Le nombre de cas chez les personnes nées à l'étranger est passé de 1224 en 2016 à 1290 en 2017, mais le taux d'incidence correspondant a diminué, passant de 15,3 à 14,7 par 100000 habitants.

Le pays de naissance a été déclaré pour $97,4 \%(n=1256)$ des personnes atteintes nées à l'étranger. Comme en 2016, les pays d'origine les plus fréquemment signalés pour ces cas étaient les Philippines ( $n=276 ; 21,4 \%)$, I'Inde ( $n=262 ; 20,3 \%)$, la Chine ( $=186 ; 14,4 \%)$, le Vietnam $(n=60 ; 4,7 \%)$ et le Pakistan $(n=46$; $3,6 \%)$.

Le statut d'immigration au moment du diagnostic de TB était connu pour $63,3 \%(n=816)$ des cas de TB signalés en 2017 chez des personnes nées à l'étranger. Parmi ces cas, $77,7 \%(n=634)$ étaient des citoyens canadiens ou des résidents permanents, $9,6 \%(n=78)$ étaient des résidents temporaires (notamment des étudiants, des visiteurs et des travailleurs) et 5,4\% $(n=44)$ étaient des réfugiés, des réfugiés au sens de la Convention ou des demandeurs d'asile. Le statut d'immigration a été déclaré comme " autre " sans plus de précisions dans 7,4\% des cas $(n=$ 60).

En 2017, I'année d'arrivée au Canada a été déclarée pour 91,0\% ( $n=1174)$ des cas de TB chez des personnes nées à l'étranger. Parmi ces cas, $36,1 \%(n=424)$ sont arrivés au cours des cinq dernières années (entre 2013 et 2017) et $17,7 \%(n=208)$ ont reçu le diagnostic de TB dans les deux années suivant leur arrivée.

\section{Table 2: Taux d'incidence de la tuberculose et nombre de cas selon l'origine et l'année, Canada,} 2007-2017

\begin{tabular}{|c|c|c|c|c|c|c|c|c|c|c|c|c|}
\hline \multicolumn{2}{|c|}{ Origine } & 2007 & 2008 & 2009 & 2010 & 2011 & 2012 & 2013 & 2014 & 2015 & 2016 & 2017 \\
\hline \multirow{2}{*}{$\begin{array}{l}\text { Non- } \\
\text { Autochtones nés } \\
\text { au Canada }\end{array}$} & Cas & 171 & 222 & 238 & 185 & 186 & 174 & 159 & 168 & 167 & 140 & 125 \\
\hline & Taux & 0,7 & 0,9 & 1 & 0,7 & 0,7 & 0,7 & 0,6 & 0,6 & 0,6 & 0,6 & 0,5 \\
\hline \multirow[t]{2}{*}{ Nés à l'étranger } & Cas & 1067 & 1064 & 1063 & 1054 & 1108 & 1112 & 1153 & 1110 & 1177 & 1224 & 1290 \\
\hline & Taux & 14,8 & 14,5 & 14,4 & 14,1 & 14,7 & 14,6 & 14,9 & 14,2 & 14,9 & 15,3 & 14,7 \\
\hline \multirow{2}{*}{$\begin{array}{l}\text { Tous les } \\
\text { Autochtones }^{\text {a }}\end{array}$} & Cas & 307 & 347 & 340 & 261 & 303 & 380 & 315 & 320 & 281 & 331 & 313 \\
\hline & Taux & 23,9 & 26,2 & 24,9 & 18,6 & 20,7 & 24,4 & 19,9 & 20 & 17,1 & 23,3 & 21,5 \\
\hline \multirow{2}{*}{$\begin{array}{l}\text { Membres des } \\
\text { Premières } \\
\text { Nations (PN) })^{a, b}\end{array}$} & Cas & 229 & 232 & 227 & 121 & 177 & 208 & 205 & 182 & 157 & 208 & 154 \\
\hline & Taux & 28,4 & 28,2 & 26,9 & 14,1 & 19,1 & 21,2 & 20,3 & 18 & 15,2 & 23,6 & 17,1 \\
\hline \multirow{2}{*}{$\begin{array}{l}\text { Membres des } \\
\text { PN vivant à } \\
\text { I'intérieur des } \\
\text { réserves }\end{array}$} & Cas & 129 & 119 & 122 & 109 & 99 & 113 & 148 & 106 & 101 & 149 & 97 \\
\hline & Taux & 29,7 & 26,8 & 27 & 23,7 & 21,2 & 23,8 & 30,8 & 21,7 & 20,4 & 33,9 & 21,7 \\
\hline \multirow{2}{*}{$\begin{array}{l}\text { Membres des } \\
\text { PN vivant à } \\
\text { l'extérieur des } \\
\text { réserves }\end{array}$} & Cas & 83 & 98 & 87 & 73 & 66 & 80 & 50 & 68 & 51 & 56 & 38 \\
\hline & Taux & 24,2 & 28 & 24,3 & 20 & 16,4 & 18,7 & 11,4 & 15,2 & 11,1 & 14,5 & 9,6 \\
\hline \multirow[t]{2}{*}{ Métis ${ }^{a}$} & Cas & 32 & 27 & 25 & 26 & 21 & 11 & 18 & 19 & 12 & 10 & 17 \\
\hline & Taux & 7,5 & 6,1 & 5,4 & 5,4 & 4,4 & 2,2 & 3,5 & 3,6 & 2,2 & 2,1 & 3,5 \\
\hline \multirow[t]{2}{*}{ Inuit ${ }^{a}$} & Cas & 46 & 88 & 88 & 114 & 105 & 161 & 92 & 119 & 112 & 114 & 142 \\
\hline & Taux & 85,2 & 160 & 157,1 & 200 & 166,7 & 251,6 & 139,4 & 177,6 & 164,7 & 170,1 & 205,8 \\
\hline \multirow{2}{*}{$\begin{array}{l}\text { Total pour le } \\
\text { Canadac }\end{array}$} & Cas & 1575 & 1642 & 1654 & 1586 & 1621 & 1700 & 1651 & 1615 & 1641 & 1750 & 1796 \\
\hline & Taux & 4,8 & 4,9 & 4,9 & 4,7 & 4,7 & 4,9 & 4,7 & 4,5 & 4,6 & 4,8 & 4,9 \\
\hline
\end{tabular}

a La Colombie-Britannique n'a pas précisé l'origine autochtone des personnes atteintes de TB pour 2016 et 2017

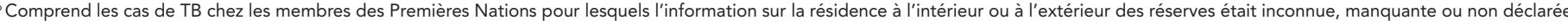
Comprend les cas de TB pour lesquels l'origine était inconnue, manquante ou non déclarée 


\section{Classification diagnostique}

En 2017, la classification diagnostique a été rapportée dans 1786 cas $(99,4 \%$ du nombre total de cas de TB). Parmi ceux-ci, 1403 (78,6\%) étaient classés comme une TB de l'appareil respiratoire et $383(21,4 \%)$, comme une TB non respiratoire. Parmi les cas de TB de l'appareil respiratoire, la forme la plus répandue était la TB pulmonaire ( $88,3 \% ; n=1239)$. Parmi les cas de TB non respiratoire, la forme la plus souvent déclarée était la TB des ganglions lymphatiques périphériques $(50,7 \% ; n=194)$. Dans les données selon l'origine, une plus grande proportion des Autochtones nés au Canada étaient atteints de TB de l'appareil respiratoire $(93,9 \%)$ que pour les non-Autochtones nés au Canada (81,5\%) et les personnes nées à l'étranger (74,3\%). Comme pour les années précédentes, la TB de l'appareil respiratoire était plus fréquente chez les hommes $(83,2 \%)$ que chez les femmes $(72,7 \%)$, de même que chez les patients de moins de 15 ans $(91,5 \%)$ que chez ceux de 15 ans et plus $(77,2 \%)$.

\section{Résultats des traitements pour 2016}

En 2016, 1750 cas de TB ont été déclarés dans le SCDCT. Parmi ceux-ci, le résultat du traitement antituberculeux a été déclaré dans $98,6 \%(n=1725)$ des cas en 2017. Pour la majorité d'entre eux $(80,2 \%)$, le traitement a été rapporté comme un succès (c'est-à-dire que le patient est guéri de la TB ou qu'il a terminé le traitement antituberculeux). Dans 7,6\% des cas, le patient est décédé avant ou pendant le traitement, et la TB aurait contribué au décès ou en serait la cause dans environ $60 \%$ de ces cas. Le traitement a été signalé comme étant en cours dans $5,3 \%$ des cas et, dans 3,0\% des cas, le patient a été transféré à l'extérieur du territoire de compétence où le traitement a été déclaré pendant que ce dernier était en cours. Les cas pour lesquels on n'a pas pu faire le suivi ou qui ont mis fin au traitement à la suite d'un événement indésirable représentaient $0,6 \%$ des résultats et les issues du traitement sont inconnues pour $2,3 \%$ des cas.

Les Inuit et les Métis affichaient les taux de réussite les plus élevés : $92,8 \%$ des patients inuit et $88,9 \%$ des patients métis ont été guéris ou ont achevé leur traitement. Les taux de réussite du traitement étaient similaires pour les membres des Premières Nations (78,3\%), les personnes nées à l'étranger $(79,1 \%)$ et les non-Autochtones nés au Canada (78,0 \%).

\section{Discussion}

En 2017, le nombre de cas de TB déclarés a augmenté de $2,6 \%$ par rapport à 2016 , et le taux d'incidence est passé de 4,8 à 4,9 pour 100000 habitants. Depuis 2014, le nombre de cas et le taux d'incidence ont tous deux affiché une croissance constante. Malgré ces augmentations des dernières années, le taux d'incidence a peu changé depuis 2007, alors qu'il était de 4,8 pour 100000 habitants. En 2017, le Nunavut a continué d'afficher le taux de TB le plus élevé au Canada, avec 265,8 cas pour 100000 habitants, soit près de 70 fois le taux national, mais la plus forte concentration de cas était en Ontario (37,6\%). Bien que le taux de TB parmi les personnes nées à l'étranger soit demeuré relativement stable au cours de la dernière décennie, cette population continue de représenter la majorité des cas en 2017 (71,8 \%). Chez les membres des Premières Nations, le taux d'incidence de la TB en 2017 (17,1 pour 100 000) a diminué par rapport à 2016 (23,6 pour 100000$)$, mais il était comparable à ceux de 2015 (15,2 pour 100 000) et de 2014 (18,0 pour 100 000). Le taux chez les Inuit au Canada en 2017 (205,8 cas pour 100000 habitants) était à son niveau le plus élevé depuis 2012 (251,6 pour 100 000). En 2017, le nombre absolu de cas signalés parmi les Inuit a également augmenté par rapport à 2016, passant de 113 à 142 cas. La majorité des cas de TB au Canada en 2017 étaient des cas de TB de l'appareil respiratoire $(78,6 \%)$, la TB pulmonaire étant la classification diagnostique la plus souvent déclarée $(69,4 \%)$. Pour la majorité des cas de TB signalés en 2016 , le traitement antituberculeux a été un succès $(80,4 \%)$, ce qui révèle que les traitements sont efficaces et que beaucoup de patients les suivent.

À partir de ces seules données de surveillance, on ne sait pas pourquoi le nombre de cas de TB a augmenté au Canada au cours des dernières années, mais plusieurs facteurs peuvent y contribuer. L'augmentation du nombre de cas et du taux d'incidence entre 2016 et 2017 reflète les augmentations du nombre de personnes nées à l'étranger et d'Inuit au cours de cette période. Bien que le taux d'incidence de la TB dans la population née à l'étranger soit demeuré stable ces dernières années, le nombre de cas a connu une croissance constante. Cela peut s'expliquer en partie par l'augmentation globale du nombre de migrants au Canada au cours des dernières années. Les personnes nées à l'étranger qui sont atteintes d'une infection tuberculeuse latente (non contagieuse) peuvent contracter une TB active plusieurs années après le processus de migration, ou encore contracter la TB pendant un voyage dans leur pays d'origine. Les conditions de vie stressantes, les barrières linguistiques et culturelles, de même que l'insécurité alimentaire et sur le plan du logement sont tous des facteurs susceptibles $d^{\prime}$ 'accroître le risque de réactivation d'une infection tuberculeuse latente après la migration (7). Les augmentations du nombre de cas de TB dans la population inuite sont aussi imputables à un risque accru de passer d'une infection tuberculeuse latente à une TB active, ou de transmission de la TB active entre deux personnes, en raison des inégalités dans l'accès aux soins de santé et dans les déterminants sociaux de la santé $(4,7)$. De plus, on a récemment signalé dans le Nord du Canada des progrès dans la détection, le diagnostic et le traitement de la TB, ce qui pourrait contribuer à une hausse de la détection des cas de TB chez les Inuit (4).

\section{Limites}

Les limites du SCDCT ont déjà été expliquées en détail (8). Le SCDCT est un système de surveillance passive qui repose sur le signalement des cas de TB active au Canada qui ont été diagnostiqués par des professionels de la santé d'un bout à l'autre du pays, qui ont été déclarés aux autorités sanitaires 
provinciales et qui, ensuite, ont été signalés à l'ASPC. Ce système peut donc présenter certaines lacunes quant à l'exhaustivité des cas signalés, ainsi qu'en raison des retards dans la déclaration des cas. Cela dit, l'OMS estime que le système canadien de surveillance affiche un taux de détection des cas de $92 \%$ (12).

Enfin, il est important de reconnaître que les données présentées dans ce rapport sont considérées comme provisoires et que, puisqu'elles sont mises à jour chaque année, elles peuvent changer dans les prochains rapports de surveillance de la TB. S'il y a des divergences entre les données résumées dans le présent rapport et les rapports provinciaux et territoriaux, le plus récent rapport provincial ou territorial devrait être utilisé, car il se peut que des données à jour soient encore attendues à l'échelon national.

\section{Conclusion}

Les données de surveillance de la TB de 2017 continuent de mettre en évidence des tendances bien connues concernant l'épidémiologie de la TB au Canada : les Canadiens autochtones ou nés à l'étranger continuent d'être représentés de manière disproportionnée et, au cours des quatre dernières années, le nombre total de cas a légèrement augmenté. La déclaration annuelle des données de surveillance de la TB au Canada est un outil important pour éclairer les efforts de prévention et de contrôle de la TB et pour suivre les progrès des initiatives visant à réduire le fardeau de la TB au Canada, l'objectif ultime étant d'éliminer complètement cette maladie.

\section{Déclaration des auteurs}

ML - Conceptualisation, Méthodologie, Logiciel, Validation, Analyse formelle, Rédaction - ébauche originale, Supervision $\mathrm{HH}$ - Conceptualisation, Logiciel, Validation, Conservation des données, Rédaction - examen et révision

$\mathrm{NH}$ - Conceptualisation, Logiciel, Validation, Conservation des données, Rédaction - examen et révision

MM - Rédaction - examen et révision, Supervision

\section{Conflit d'intérêt}

Aucun.

\section{Remerciements}

L'Agence de la santé publique du Canada tient à remercier les employés des programmes provinciaux et territoriaux suivants pour leur contribution et leur participation au SCDCT :

Brenda P. Earles, ministère de la Santé et des Services

communautaires, Direction générale de la santé de la population, Terre-Neuve-et-Labrador

Beth Halfyard, ministère de la Santé et des Services

communautaires, Direction générale de la santé de la population, Terre-Neuve-et-Labrador

Shamara Baidoobonso, ministère de la Santé et du Mieux-être, Île-du-Prince-Édouard
Stacey Burns, ministère de la Santé et du Mieux-être, Île-du-Prince-Édouard

Constance Cheverie, ministère de la Santé et du Mieux-être, Île-du-Prince-Édouard

Beverly A. Billard, ministère de la Santé et du Mieux-Être, Nouvelle-Écosse

Suzanne Savoie, ministère de la Santé, Nouveau-Brunswick Hanan Smadi, ministère de la Santé, Nouveau-Brunswick Isabelle Rouleau, Direction régionale de santé publique de Montréal, Québec

Michael Whelan, Santé publique Ontario

Carla Loepky, ministère de la Santé, Manitoba

Luiz C. Mostaço-Guidolin, ministère de la Santé, Manitoba

Helen Bangura, ministère de la Santé, Saskatchewan

Valerie Mann, ministère de la Santé, Saskatchewan

Assaad Al-Azem, Prévention et contrôle de la TB, Saskatchewan

Sandy Cockburn, Services de santé de l'Alberta

David Cao, Services de santé de l'Alberta

Fay Hutton, Centre de contrôle des maladies de la Colombie-Britannique

Sukhman Thind, Centre de contrôle des maladies de la

Colombie-Britannique

David Roth, Centre de contrôle des maladies de la

Colombie-Britannique

Jannie Leung, Centre de contrôle des maladies de la

Colombie-Britannique

Meadow Allen, Centre de lutte contre les maladies

transmissibles, Yukon

Lori Strudwick, Centre de lutte contre les maladies

transmissibles, Yukon

Caroline NewBerry, ministère de la Santé et des Services sociaux, Territoires du Nord-Ouest

Heather Hannah, ministère de la Santé et des Services sociaux, Territoires du Nord-Ouest

Karen Hollett, ministère de la Santé et des Services sociaux, Territoires du Nord-Ouest

Andrea Schertzer, ministère de la Santé, Nunavut

\section{Financement}

Cet article a reçu l'appui financier de l'Agence de la santé publique du Canada dans le cadre de son mandat principal.

\section{Références}

1. World Health Organization. Global tuberculosis report 2018. Geneva (CH): WHO; 2018. https://www.who.int/tb/ publications/global_report/en/

2. World Health Organization. Framework towards tuberculosis elimination in low incidence countries. Geneva $(\mathrm{CH})$ : WHO; 2014. www.who.int/tb/publications/elimination_framework/ en/

3. Vachon J, Gallant V, Siu W. La tuberculose au Canada, 2016. Relevé des maladies transmissibles au Canada. 2018;43(3/4):85-91. DOI 
4. Patterson M, Finn S, Barker K. Lutter contre la tuberculose chez les Inuit au Canada. Relevé des maladies transmissibles au Canada. 2018;44(3/4):92-5. DOI

5. Statistique Canada. Les peuples autochtones au Canada : faits saillants du Recensement de 2016. Ottawa (ON) : Statistique Canada; oct 25, 2017. https://www150.statcan. gc.ca/n1/daily-quotidien/171025/dq171025a-fra.htm

6. Statistique Canada. Immigration et diversité ethnoculturelle : faits saillants du Recensement de 2016. Ottawa (ON) : Statistique Canada; oct 25, 2017. https://www150.statcan. gc.ca/n1/daily-quotidien/171025/dq171025b-fra.htm

7. Agence de la santé publique du Canada. Un message de I'administratrice en chef de la santé publique du Canada. Ottawa (ON) : ASPC; 2018. https://www.canada.ca/fr/ sante-publique/organisation/publications/rapports-etatsante-publique-canada-administrateur-chef-sante-publique/ eliminer-tuberculose.html

8. Agence de la santé publique du Canada. La tuberculose au Canada 2012. Ottawa (ON) : Ministre des Travaux publics et des Services gouvernementaux Canada; 2015. https://www. canada.ca/fr/sante-publique/services/maladies-infectieuses/ tuberculose-canada-2012.html

9. Agence de la santé publique du Canada. Menzies D, Wong $T$, rédacteurs. Normes Canadiennes pour la lutte antituberculeuse 7e édition.ASPC; 2014. https://www. canada.ca/fr/sante-publique/services/maladies-infectieuses/ normes-canadiennes-lutte-antituberculeuse-7e-edition.html

10. Statistique Canada. Tableaux de données, Recensement de 2016. Citoyenneté (5), lieu de naissance (272), statut d'immigrant et période d'immigration (11), âge (12) et sexe (3) pour la population dans les ménages privés du Canada, provinces et territoires, régions métropolitaines de recensement et agglomérations de recensement, Recensement de 2016 - Données-échantillon (25\%). № au catalogue : 98-400-X2016184. Ottawa (ON) : Statistique Canada; 2016. https://www12.statcan.gc.ca/censusrecensement/2016/dp-pd/dt-td/Ap-fra.cfm?LANG=F\&APA $\mathrm{TH}=3 \& D E T A I L=0 \& D I M=0 \& F L=A \& F R E E=0 \& G C=0 \& G I D=0$ $\& G K=0 \& G R P=1 \& P I D=110525 \& P R I D=10 \& P T Y P E=109445 \&$ $\mathrm{S}=0 \& \mathrm{SHOWALL}=0 \& \mathrm{SUB}=0 \&$ Temporal $=2017 \& \mathrm{THEME}=120$ $\& \mathrm{VID}=0 \& \mathrm{VNAMEE}=\& \mathrm{VNAMEF}=$

11. Morency JD, Caron-Malenfant E, Coulombe S, Langlois S. Projections de la population et des ménages autochtones au Canada, 2011 à 2036. No au catalogue : 91-552. Ottawa (ON): Statistique Canada; 2015. https://www150.statcan. gc.ca/n1/pub/91-552-x/91-552-x2015001-fra.htm

12. World Health Organization. Global tuberculosis report 2017. Geneva (CH): WHO; 2017. https://www.who.int/tb/ publications/global_report/gtbr2017_main_text.pdf

\section{Annexe : Liste des tableaux supplémentaires}

Pour obtenir les tableaux supplémentaires, veuillez en faire la demande à : phac.tb.surveillance.aspc@canada.ca

Tableau supplémentaire 1 : Nouveaux cas de tuberculose active et de retraitement déclarés et taux d'incidence par 100000 habitants (tous les cas) Canada et provinces et territoires : 2007-2017

Tableau supplémentaire 1M : Nouveaux cas de tuberculose active et de retraitement déclarés et taux d'incidence par 100000 habitants (hommes) Canada et provinces et territoires : 2007-2017

Tableau supplémentaire 1F : Nouveaux cas de tuberculose active et de retraitement déclarés et taux d'incidence par 100000 habitants (femmes) Canada et provinces et territoires : 2007-2017

Tableau supplémentaire 2 : Nouveaux cas de tuberculose active et de retraitement déclarés et taux d'incidence par 100000 habitants (selon le groupe d'âge) - Canada : 2007-2017

Tableau supplémentaire 3 : Nouveaux cas de tuberculose active et de retraitement déclarés et taux d'incidence par 100000 habitants (selon le groupe d'âge) - Canada et provinces et territoires : 2017

Tableau supplémentaire 4 : Nouveaux cas de tuberculose active et de retraitement déclarés et taux d'incidence par 100000 habitants (selon l'origine) - Canada et provinces et territoires : 2017

Tableau supplémentaire 5 : Taux d'incidence pour la tuberculose par 100000 habitants (selon l'origine) - Canada : 2007-2017

Tableau supplémentaire 6 : Nouveaux cas de tuberculose active et de retraitement déclarés et taux d'incidence par 100000 habitants (selon la principale classification diagnostique) - Canada : 2007-2017

Tableau supplémentaire 7 : Nouveaux cas de tuberculose active et de retraitement déclarés et taux d'incidence par 100000 habitants (selon la principale classification diagnostique) - Canada et provinces et territoires : 2017

Tableau supplémentaire 8 : Résultats des traitements - Canada et provinces et territoires : 2016 